Revista

Multi-Ensayos

Vol. 5, $\mathrm{N}^{\circ} 10$

ISSN: 2412-3285

https://multiensayos.unan.edu.ni

DOI: https://doi.org/10.5377/multiensayos.v5i10.8870

\title{
Diversas metodologías en los procesos de enseñanzas de aprendizajes
}

\section{Various methodologies in learning teaching processes}

Sandy Maritza Ramírez Castillo ${ }^{1}$

Recibido: 02 de junio de 2019. Aceptado: 30 de junio de 2019

\section{RESUMEN}

Efectuar diversas metodologías en las enseñanzas de aprendizajes en la educación superior, permite que los estudiantes se sientan motivados, desarrollen habilidades en la oralidad, participación activa. Se comparte experiencia sobre la aplicación de las diversas metodologías, con el grupo de II año de la carrera de Ciencias Políticas y Relaciones Internacionales, UNAN-MANAGUA-FAREM-Estelí, cuyos resultados se toman como logros buen rendimiento académico, participación activa, desarrollar habilidades en la oralidad, apropiación de la materia. Como dificultades el uso continuo del celular, falta de respeto entre las estudiantes, llegadas tarde de clase, ausencias continuas.

Palabras claves: metodología; interés; estrategias; enseñanza.

\section{ABSTRACT}

Performing various methodologies in learning teachings in higher education, allows students to feel motivated, develop skills in orality, active participation. Experience is shared on the implementation of the various methodologies, with the group of II year of the career of Political Science and International Relations, UNAN-MANAGUA-FAREM-Estelí, which results are taken as good achievements academic performance, active participation, developing skills in orality, appropriation of the subject. As difficulties the continuous use of their cell phone, lack of respect among students, late arrivals of class, continuous absences.

Keywords: methodology; interest; strategies; teaching.

1 Docente UNAN-Managua/FAREM-Estelí. Correo electrónico: .

(c) 2019 Revista Multi-Ensayos. 


\section{INTRODUCCIÓN}

El tema a tratar para el presente ensayo consiste "Diversas metodologías en los procesos de enseñanzas de aprendizajes universitarios", el objetivo de abordar esta temática es compartir como docente el resultado que llevó implementar nuevas metodología con las diferentes carreras de la Universidad Nacional Autónoma de UNAN- MANAGUA/FAREM-Estelí.

En la primer temática se aborda sobre términos conceptuales como métodos y metodología, métodos activos de enseñanzas, la importancia de utilizar diferentes formas de metodología en los procesos de enseñanzas de aprendizaje. La segunda temática se aborda la experiencia con el grupo de II año de Ciencias Política y Relaciones Internacionales del turno Matutino, dando a conocer los resultados que se obtuvieron al aplicar diferentes metodologías y estrategias en el salón de clase.

Por otro lado se hace un análisis de esos resultados, identificando para ello los logros, aquellas dificultades que se presentaron, viendo así todos aquellos puntos negativos, desde el punto de vista para el docente como para el estudiante, así como identificar si hubo deserción y sus causales.

\section{DESARROLLO}

\section{Método y metodología:}

Según Picardo Joao (2005) La ciencia es un tipo particular y específico de conocimiento. Para lograr un conocimiento de tal naturaleza, o sea, para hacer ciencia, es preciso seguir determinados procedimientos que nos permitan alcanzar el fin que procuramos: no es posible obtener un conocimiento racional, sistemático y organizado actuando de cualquier modo; es necesario seguir un método, un camino que nos aproxime a esa determinada meta.

El método científico es el procedimiento o conjunto de procedimientos que se utilizan para obtener conocimientos científicos, el modelo de trabajo o pauta general que orienta la investigación. El estudio del método o de los métodos, si se quiere dar al concepto un alcance más general se denomina metodología, y abarca la justificación y la discusión de su lógica interior, el análisis de los diversos procedimientos concretos que se emplean en las investigaciones y la discusión acerca de sus características, cualidades y debilidades.

Sin embargo, se suele utilizar la palabra metodología en sentidos diferentes, opuestos a veces al anterior: se habla así de "metodología de la investigación" para hacer referencia a los pasos y procedimientos que se han seguido en una indagación determinada, para designar los modelos concretos de trabajo que se aplican en una determinada disciplina o especialidad y también para hacer referencia al conjunto de procedimientos y recomendaciones que se transmiten al estudiante como parte de la docencia en estudios superiores. También suelen designarse como métodos los estilos de trabajo peculiares de cada disciplina (por ejemplo: "el método antropológico") y las formas particulares de investigación que se 
utilizan para resolver problemas específicos de indagación, como cuando se habla del "método cualitativo", el "método experimental" o el "método estadístico".

El método se refiere directamente a la lógica interior del proceso de descubrimiento científico, y a él le corresponde no solamente orientar la selección de los instrumentos y técnicas específicos de cada estudio, sino también, fundamentalmente, fijar los criterios de verificación y demostración de lo que se afirme en la investigación.

\section{Métodos activos de enseñanza profesional}

Son las formas y procedimientos que sitúan al alumno en una posición activa, al incrementar su participación en el proceso pedagógico profesional, y ofrecerle a través de esa participación activa, las vías para la adquisición de conocimientos y el desarrollo de hábitos y habilidades generalizadoras, que los capaciten para enfrentar exitosamente futuras problemáticas en el campo profesional para el cual se preparan, y para solucionar los proyectos y tareas planteadas por el docente.

De lo anterior se puede apreciar que la metodología implica entonces al proceso que se da durante el desarrollo de la enseñanza educativa para con los estudiantes, con ello se permite a que los estudiantes sean más activos en cuanto a su participación y dicho sea de paso apropiarse de la materia. (Picardo Joao, 2005)

\section{¿Qué Entendemos por Estrategia Didáctica?}

El término estrategia se utiliza con preferencia por responder mejor a un enfoque interactivo y ecosistémico. La realidad social, educativa, creativa no son lineales, ni rígidas, ni estáticas, sino por el contrario se caracterizan por ser complejas, adaptativas, cambiantes, interactivas, deudoras de entornos y contextos socioculturales. Es por ello que el concepto de estrategia responde mejor a nuestros propósitos, entendida como procedimiento adaptativo o conjunto de ellos por el que organizamos secuenciadamente la acción para lograr el propósito o meta deseado. Las estrategias nos acompañan siempre haciendo de puente entre metas o intenciones y acciones para conseguirlos. (Torre \& Violant, 1993)

De lo anterior es importante señalar que la estrategia se plantea desde que se inicia a realizar el plan didáctico del inicio de cada semestre, lo que comprende de forma detallada cuáles son las metas que se tiene como docente haciendo uso de diferentes estrategias para un mejor desarrollo de la clase a abordarse.-

\section{Una Metodología de Desarrollo para Investigar con creatividad}

Uno de los objetivos de especial relevancia en el transcurso de los años de docencia universitaria, ha sido: describir y determinar el impacto y grado de satisfacción del alumnado ante la utilización de estrategias creativas diferentes, variando las asignaturas, la enseñanza, el profesorado. 
Para Torre \& Violant (1993) caracterizan dichas estrategias por la flexibilidad en la planificación, la adaptación contextual, la creación de un clima distendido y gratificante, roles participativos e interactivos entre el alumnado y éste con el profesorado, productividad o realizaciones personales, alto grado de satisfacción, conciencia de autoaprendizaje. Estos rasgos tienen que ver con las vertientes claves de la creatividad: la persona, el proceso, el ambiente y el resultado en su doble vertiente productiva y de satisfacción personal. Como vemos se trata de establecer los efectos que puedan tener las estrategias didácticas creativas entendidas en los términos indicados.

\section{Métodos de enseñanza UDLA}

Para Maureira Miranda \& Alfaro Lay (2015) existen tres métodos de enseñanza que se aplican para el desarrollo de sus procesos formativos:

- Método tradicional o de control docente: A través de este método el docente entrega a los estudiantes diversos saberes (conceptuales, procedimentales y actitudinales). Para ello, utiliza clases expositivas y demostraciones, complementadas por libros de texto.

- Método facilitador de la comprensión o de control del docente y el estudiante: $A$ través de este método el docente ayuda a los estudiantes a construir un significado que les permita comprender las ideas y procesos clave; los guía en discusiones en torno a problemas complejos, textos, casos, proyectos o situaciones, generando instancias para el cuestionamiento, el establecimiento de pruebas y la reflexión sobre procesos.

- Método de revisión del desempeño o de control del estudiante: A través de este método el docente evalúa el trabajo autónomo de los estudiantes mediante la demostración o simulación de saberes profesionales vinculados al mundo laboral.

Para ello, el docente comunica a los estudiantes los resultados de aprendizaje relacionados con el desempeño que evaluará y supervisa, a través de instancias de retroalimentación, el desarrollo de las habilidades en el contexto de oportunidades de aprendizaje. Un claro ejemplo de este método se observa en las asignaturas prácticas, pues en ellas se aplican estrategias vinculadas con el aprendizaje en servicio o de intervención comunitaria.

\section{Experiencia con la aplicación de diversas metodologías}

Una vez que se abordó diversos conceptos relacionados a la metodología, en este apartado se comparte la experiencia que se tuvo en la aplicación de diversas metodologías en el grupo de segundo año de la carrera de Ciencias Políticas y Relaciones Internacionales del turno matutino:

La materia que se abordó para el grupo de segundo año de la carrera de Ciencias Políticas, fue Sistema Político, que contiene diversas unidades entre esas, Conceptos Fundamentales en el estudio de los Sistemas Políticos, Estado, Gobernabilidad democrática, Legitimidad, Eficacia, etc. 
El primer día de clase como era un grupo al que se le había facilitado otra asignatura en el semestre anterior, no se requería de presentación personal, sin embargo como una introducción a la materia, se aplicó como estrategia de enseñanza de aprendizaje la sopa de letras que consiste que cada grupo asignado identificará una letra y la definiera, haciendo así una definición personal de cada grupo, compartiéndola.

Igualmente en el transcurso del semestre se desarrollaron diferentes estrategias tales como:

- El uso del programa (podría ser un programa de radio, tv, etc.), que consiste asignarle una temática a cada grupo, teniendo cada integrante un rol, se escogió una presentadora y los demás especialista de la materia, se les dio tiempo para que estudien dicha temática, dándoles la libertad de acondicionar el salón con diferentes tipos de materiales de apoyo, utilizando así su creatividad. Los demás estudiantes iban hacer preguntas haciendo llamadas al programa, y los especialistas tenían que contestar a todas aquellas inquietudes que tenían los televidentes. Se les daba un tiempo de 30 minutos para cada grupo.

- Ponencia de diversas temáticas: Este consiste en escoger de forma aleatoria a un expositor de cada grupo, al que se le asignaba una temática distintas de cada grupo, preparándose para dar un ponencia sobre dicho tema, dándole un tiempo de 20 minutos y un espacio de 10 minutos las preguntas y respuestas. Para este tipo de estrategia de enseñanza de aprendizaje no se requiere ningún tipo de material, cada expositor iba a dar a conocer su tema sentados, sólo abordar sin apoyo de ningún documento. Al final se hace una aclaración sobre todas aquellas dudas que se quedaron en la ponencia, y posteriormente la aplicación de una prueba sistemática para evaluar los conocimientos adquiridos con esta temática.

- Ponencias de los grupos asignados (expositor y panelistas): Este tipo de metodología, consiste en orientarle a cada grupo temáticas diferentes; escogiendo de forma aleatoria a un exponente y 3 panelistas. El exponente preparaba su material de apoyo para exponer su temática y los panelistas se debían de preparar para contestar las preguntas que hacían los demás estudiantes a través de un papelito. Al final se explicó todas aquellas dudas que tenían los estudiantes, y se aplicó prueba escrita para evaluar los conocimientos adquiridos.

- El uso de la ponencia oratoria: El docente expone la temática, haciendo uso como material de apoyo el data show, dando un espacio de preguntas y respuestas y al final se hizo una prueba sistemática escrita para evaluar los conocimientos adquiridos por los estudiantes.

- El uso del juego del saber: Este tipo de metodología consiste en realizar una clase práctica en leer, analizar y contestar 10 preguntas que deben de contestar cada grupo, presentando documentación en tiempo y forma asignado, dándoles tiempo para su debido estudio el que fue contestada a través de participación aleatoria y de forma oral, ubicando a cada estudiante en una silla de tal manera que cada silla representada a cada grupo al estudiante que era llamado y contestaba participaba otro de su mismo grupo, hasta se abarcará las preguntas. Los estudiantes que no alcanzaron participar al final se realiza una prueba sistemática escrita para evaluar los conocimientos adquiridos en este tipo de enseñanza. 
Con la aplicación de las diversas de metodologías se detectaron fortalezas, logros, y debilidades, las que se describen a continuación:

\begin{tabular}{|c|c|c|}
\hline & & \\
\hline $\begin{array}{l}\text { - La implementación de diversas } \\
\text { metodologías. } \\
\text { - El interés que tiene la mayoría } \\
\text { de los estudiantes en tener } \\
\text { conocimientos sobre la materia } \\
\text { que se aborda. } \\
\text { - La participación activa de } \\
\text { involucrarse en las diversas } \\
\text { metodologías aplicadas. } \\
\text { Establecer un ambiente de } \\
\text { confianza. }\end{array}$ & 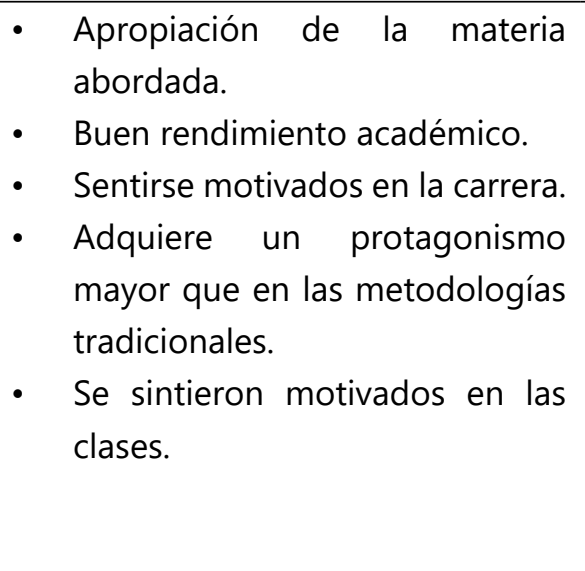 & $\begin{array}{l}\text { continuo de celular, se } \\
\text { umpía las clases. } \\
\text { y orden en la participación, } \\
\text { quieren participar a la vez. } \\
\text { de interés para algunos } \\
\text { antes que tuvieron mucha } \\
\text { encia. } \\
\text { iste respeto mutuo entre } \\
\text { udiantes. }\end{array}$ \\
\hline
\end{tabular}

Es importante señalar que el aplicar las diversas metodologías, permitió motivar a que los estudiantes desarrollaran un mayor interés en seguir con su carrera. Cabe señalar que la enseñanza creativa se caracteriza precisamente por ser activa, motivadora, dinámica, implicativa. "El aprendizaje creativo hace referencia al conocimiento construido con la implicación activa del sujeto, desde su planificación hasta su internalización, caracterizado por la motivación intrínseca, estar centrado en el discente, carácter abierto del proceso y la autoevaluación" (Torre \& Violant, 1993).

Como una forma de evaluar esta asignatura, la docente pide opiniones a los estudiantes, ya que eso permite identificar en que se falló y si tuvo éxito la implementación de diversas metodologías. La mayoría de los estudiantes, manifestaron sentirse interesados en la materia, y satisfechos de haber aprendido las temáticas abordadas y de la importancia que implica tener conocimientos sobre sistemas políticos.

\section{CONCLUSIÓN}

La Aplicación de diversas metodologías permitió que los estudiantes, se sintieran motivados, se apropien de la materia, tener una participación activa, desarrollen habilidades de oralidad.

La motivación es vital para incentivar a los estudiantes la importancia de participar y que poco a poco se vayan apropiando de la materia.

El respeto mutuo entre sus compañeros y con el docente como una de las reglas de funcionamiento dentro del aula de clase.

Es significativo para el desarrollo de este tipo de asignaturas el establecimiento de un ambiente de confianza donde el estudiante no se sienta inhibido a la hora de participar. 
La puesta en práctica de estrategias didácticas y nuevas metodologías genera en los estudiantes el deseo de aprender con calidad y de inclusive utilizar los celulares de manera creativa.

\section{REFERENCIAS}

Maureira Miranda, A., \& Alfaro Lay, A. (2015). Guía de métodos y estrategias de enseñanzas y aprendizajes. Santiago: Avda. Manuel Montt 948.

Picardo Joao, O. (2005). Diccionario Pedagógico. El Salvador C.A: Educativa, Colegio García Flamenco. Torre, S., \& Violant, V. (1993). Estrategias Creativas en las enseñanzas universitaria. 